\title{
Preface for the SYNT
}

\section{Roderick Bloem ${ }^{1} \cdot$ Paulo Tabuada ${ }^{2}$}

Published online: 30 November 2019

๑) Springer-Verlag GmbH Germany, part of Springer Nature 2019

Synthesis from formal specifications is a programming paradigm that recruits the insight and creativity of programmers for the aspect of programming that needs it the most: specifying what the program should do. In contrast, the question of how a program meets its requirements is left to a synthesis algorithm. Synthesis can thus be regarded as programming at a higher level of abstraction where specifications are compiled into machine code.

The objective of this special issue is to provide a window into the rapidly evolving field of synthesis. Many, but not all, of the papers were originally presented at SYNT 2018, the seventh workshop on synthesis that took place on July 18th, 2018, in Oxford, co-located with the 2018 Federated Logic Conference (FLOC).

Seventeen papers were submitted for possible publication in this special issue and after a rigorous review process, ten papers were selected for publication. These papers cover different aspects of synthesis including applications to programming and to the control of physical systems, and a combination of theoretical advances as well as practical algorithmic and performance improvements.

The guest editors wish to thank all the authors for their submissions, the large number of reviewers who carefully and timely scrutinized and evaluated the many papers submitted, Acta Informatica's Editor-in-Chief, Christel Baier, for her availability and guidance throughout this process, and the Journal Editorial Office Assistant Roseline Periyanayagam for her invaluable help.

Publisher's Note Springer Nature remains neutral with regard to jurisdictional claims in published maps and institutional affiliations.

Roderick Bloem

roderick.bloem@iaik.tugraz.at

$\triangle$ Paulo Tabuada

tabuada@ee.ucla.edu

1 Institute of Applied Information Processing and Communications, Graz University of Technology, Graz, Austria

2 Department of Electrical and Computer Engineering, University of California at Los Angeles, Los Angeles, USA 